\title{
Venlafaxine-induced serotonin syndrome with relapse following amitriptyline
}

\author{
Nicholas K Perry
}

\begin{abstract}
A case of venlafaxine-induced serotonin syndrome is described with relapse following the introduction of amitriptyline, despite a 2-week period between the discontinuation of one drug and the commencement of the other. Electroencephalography may play an important part in diagnosis. With the increasing use of selective serotonin re-uptake inhibitors, greater awareness of the serotonin syndrome is necessary. Furthermore, the potential for drug interactions which may lead to the syndrome needs to be recognised.
\end{abstract}

(Postgrad Med f 2000;76:254-256)

Keywords: serotonin syndrome; venlafaxine; amitriptyline; adverse drug reaction

The serotonin syndrome, a combination of neuromuscular symptoms, alteration in mental status and derangement of autonomic function occurs as a result of excess serotonin in the nervous system. It is seen most frequently in those patients receiving a combination of drugs that enhance serotonin central nervous system activity. It can be fatal.

\section{Case report}

A 75 year old man was admitted to a local psychiatric hospital with worsening depression and self-neglect. No other significant medical history was known. His medication comprised oxitropium and becloforte inhalers for wellcontrolled asthma.

On examination he appeared malnourished and withdrawn. His mood was low. There were no other relevant physical findings. A full blood count was normal apart from a neutropenia $\left(0.8 \times 10^{9} / 1\right)$. The blood film demonstrated irregular red blood cells. Urea and electrolytes, creatinine, liver function tests, gamma glutamyl transferase and thyroid function were all within normal limits.

Sertraline (a selective serotonin re-uptake

Department of Medicine for the Elderly, Poole Hospital NHS Trust, Longfleet Road, Poole, Dorset BH15 2JB, UK

Correspondence to: Dr Perry

Submitted 3 February 1999 Accepted 10 September 1999 trying to walk. Significant postural hypotension was recorded (blood pressure $130 / 80 \mathrm{mmHg}$ lying, $95 / 50 \mathrm{mmHg}$ standing). At this time his serum sodium concentration, having been normal on admission, had fallen to $123 \mathrm{mmol} / \mathrm{l}$. The patient was transferred to our unit for further assessment.

Examination revealed cachexia and a withdrawn state. He appeared to be moderately confused and slightly dysarthric. Frequent myoclonic jerks involving all limbs were noted. A pout reflex was present. Muscle tone appeared normal and there were no other abnormal neurological signs. Significant postural hypotension was confirmed. No fever was recorded. Further biochemical analysis suggested a syndrome of inappropriate antidiuretic hormone (SIADH) secretion. The serum sodium was $127 \mathrm{mmol} / 1$ with a plasma osmolality of $269 \mathrm{mOsmol} / \mathrm{kg}$ and urine osmolality of $852 \mathrm{mOsmol} / \mathrm{kg}$. A 'spot' urinary sodium was $46 \mathrm{mmol} / \mathrm{l}$. Creatine kinase levels, a short synacthen test, and prostatic serum antigen levels were within normal limits. A myeloma screen and HIV test were negative. A full blood count was normal apart from a persisting neutropenia. Erythrocyte sedimentation rate was 41 $\mathrm{mm} / \mathrm{h}$. A bone marrow aspiration examination was non-diagnostic, although early myelodysplasia was considered possible. A chest X-ray showed no relevant abnormality and an abdominal ultrasound scan was normal. A computer tomography (CT) head scan showed sparse low attenuation around the periventricular white matter consistent with ischaemic change but no other relevant abnormality. A cerebrospinal fluid study showed an acellular fluid with normal protein and glucose levels.

A fluid restriction regime was implemented and although a normalisation in the serum sodium concentration was seen after 5 days, there was no clinical improvement. An electroencephalogram (EEG) was performed (fig 1), by which time the patient had been taking venlafaxine for 5 weeks.

Since the cause of the patient's illness remained undetermined, venlafaxine was discontinued and over a 6-day period the myoclonic jerks disappeared and his confusion improved. He was seen to be able to walk steadily and no further postural hypotension could be demonstrated. A second EEG performed 10 days after venlafaxine withdrawal showed significant improvement.

Because of his persisting low mood 2 weeks after discontinuing venlafaxine, amitriptyline was commenced at a dosage of $50 \mathrm{mg}$ bid. Over onset of confusion was noted and by the end of the third week a general deterioration had become apparent with a tendency to fall when 


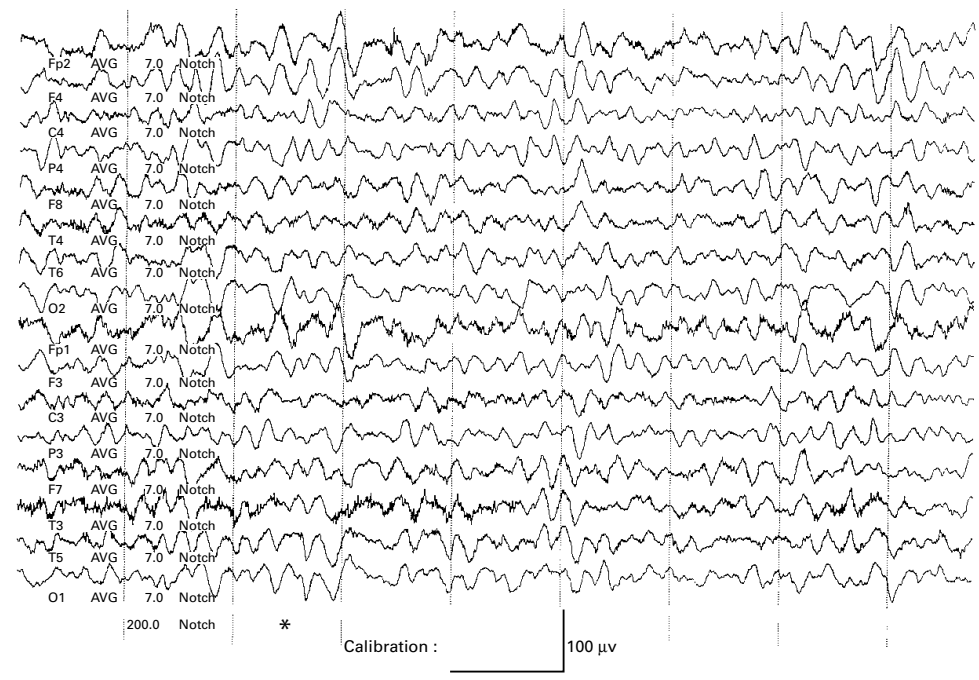

Figure 1 EEG showing absence of normal alpha rhythm and presence of slow wave activity*.

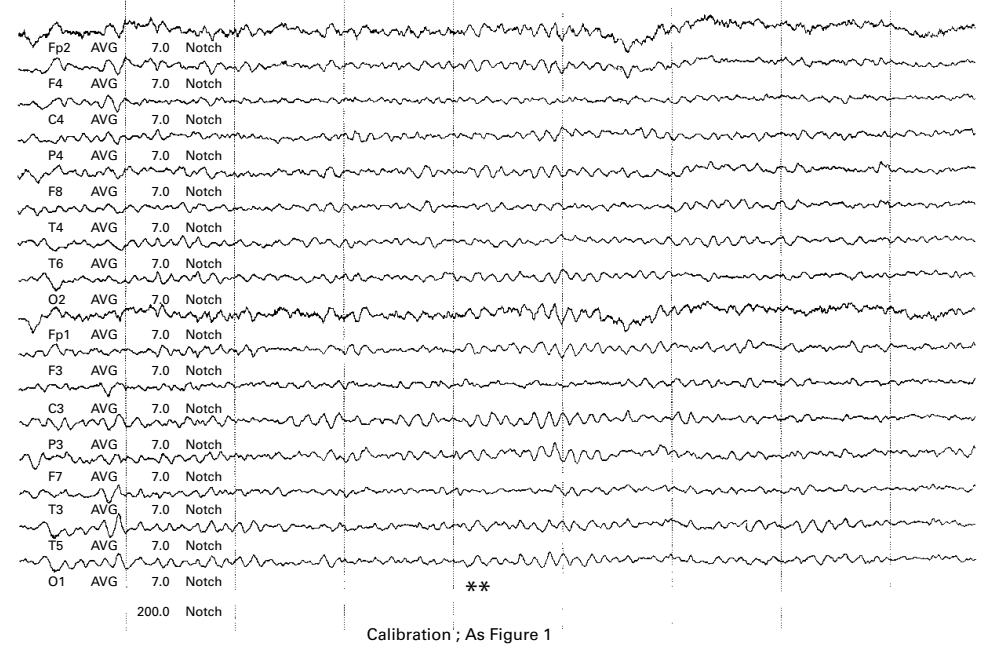

Figure 2 EEG showing return of alpha rhythm ${ }^{\star *}$.

the following 48 hours a deterioration was seen with a recurrence of myoclonic jerks and confusion. At this stage his serum sodium concentration was within normal limits. Amitriptyline was discontinued after 3 days and over the following 8 days the clinical state improved once more with abatement of myoclonic jerking and no further confusion.

A third EEG (fig 2) performed 8 days after discontinuation of amitriptyline showed further improvement. The patient was eventually transferred back to the psychiatric unit where his physical state continued to improve and some lifting of his depression was seen.

\section{Discussion}

The serotonin syndrome is an increasingly recognised and occasionally fatal disorder. It should be considered in any patient receiving serotonergic medication who demonstrates altered cognitive behaviour function, autonomic nervous system dysfunction, or neuromuscular abnormalities.

Diagnostic criteria have been developed for the serotonin syndrome (box 1). ${ }^{1}$ The syndrome typically occurs within hours or days of

\section{Box 1: Diagnostic criteria for the serotonin syndrome}

Coincident with the addition of or an increase in dosage of a known serotonergic agent to an established medication regimen, at least three of the following clinical features must be present:

- Mental state changes: agitation, confusion, restlessness, coma

- Motor system changes: myoclonus, hyperreflexia, incoordination, rigidity

- Autonomic instability: nausea, diarrhoea, vomiting, shivering, tachycardia, postural hypotension, low grade fever

Other aetiologic causes (eg, infectious, metabolic, substance abuse, or withdrawal) have been ruled out

the addition or dosage increment of a serotomimetic drug to a medication regime that already includes serotonin-enhancing drugs. Alternatively, the premature initiation of a serotomimetic agent following the recent discontinuation of a serotonin-enhancing drug with a long half-life may produce the syndrome.

The majority of case reports of serotonin syndrome have occurred with SSRIs when used alone or more usually in combination with monoamine-oxidase inhibitors (MAOIs). ${ }^{2}$ The importance of an abstinence period of at least 2 weeks before SSRI-type drugs can be administered after discontinuation of any of the MAOIs is well known. This time is required for the synthesis of functional monoamine-oxidase enzyme, thereby restoring normal amine metabolism and avoiding excessive serotonergic effects. Conversely, a period of 2 weeks between stopping an SSRI and starting MAOI treatment is usually sufficient, with the exception of fluoxetine when a wash-out period of 5 weeks is recommended. These wash-out periods after SSRI discontinuation are based on half-lives of these drugs (at least five half-lives) and are minimum recommendations. ${ }^{3}$

Perhaps less well recognised is that any drug with serotomimetic activity (box 2) may potentially cause a serotonin syndrome when serotonin transmission has been enhanced by concomitant or recently withdrawn serotomimetic therapy.

The patient described did not appear to develop the serotonin syndrome following 11 days on sertraline, possibly because this time was too short for such an adverse effect to be seen. It is, however, more likely that the addition of venlafaxine only 48 hours after discontinuing sertraline (half-life $26 \mathrm{~h}$ ) with a dosage increment after 12 days, led to the development of the syndrome, the first signs of which were noted after 14 days therapy.

There have been no previous case reports of a relapsed serotonin syndrome induced by amitriptyline despite a 2-week wash-out period off venlafaxine. The half-life of venlafaxine and its active metabolite is thought to be around 11 hours in the elderly, suggesting a minimum wash-out period of $55 \mathrm{~h}$. Since in the case described 2 weeks had elapsed, this would suggest a separate adverse serotomimetic effect of 


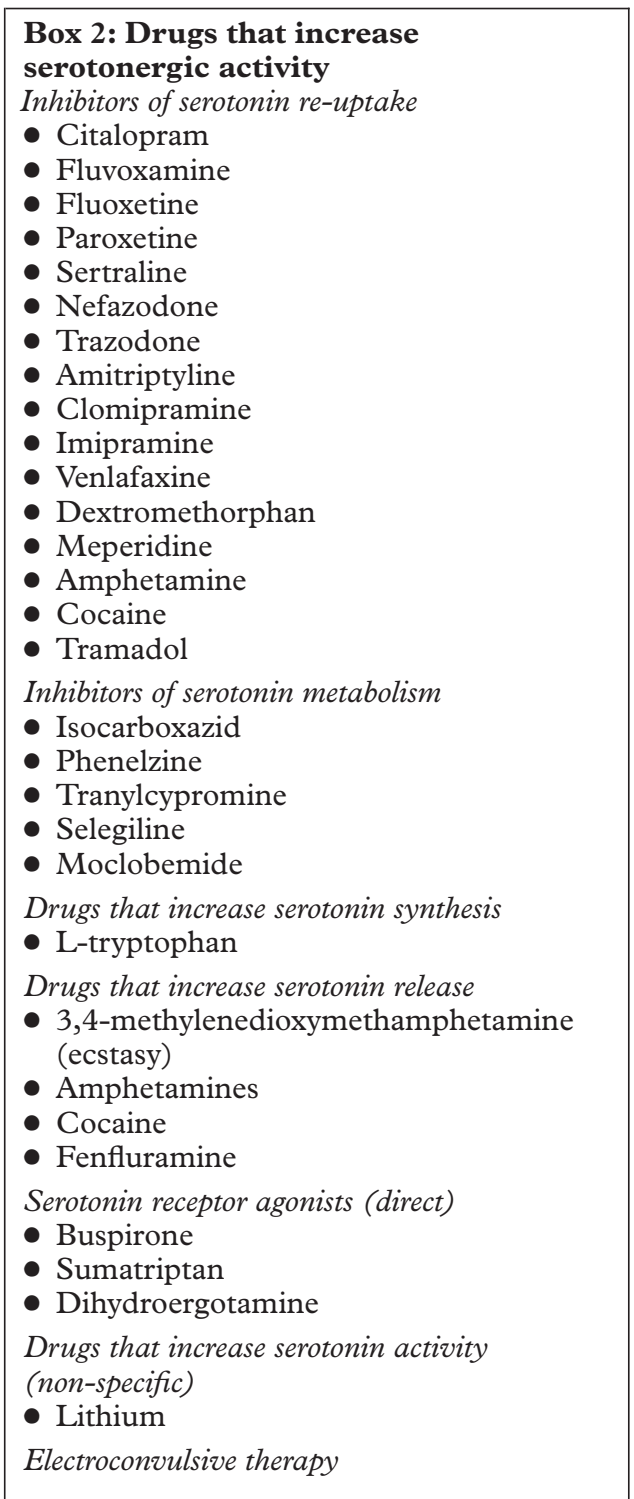

amitriptyline, although an inadequate washout period cannot be excluded.

Other case reports of the serotonin syndrome have noted various EEG abnormalities, ${ }^{45}$ including delta range activity, slow waves, spike and waves, polyspike and waves and triphasic waves. The first EEG (fig 1) shows abnormal slow wave activity and absence of normal alpha rhythm. Neither triphasic waves (commonly found in metabolic encephalopathy) nor periodic complexes (suggestive of CreutzfeldtJacob disease) are present. Following venlafaxine withdrawal, less slow wave activity is apparent and a return to an alpha rhythm is seen (fig 2).

SIADH is a well recognised feature of SSRI therapy and has recently been reported to occur with venlafaxine. ${ }^{6}$ Whilst contributing to this patient's illness it is suspected that SIADH did not play a major role, given the continuation of his encephalopathy despite normalisation of the serum sodium concentration. Furthermore, postural hypotension is specifically not a feature of SIADH but is recognised to occur in the serotonin syndrome. Also of note is that the serum sodium concentration was within normal limits during the amitriptyline-induced relapse.

The management of the serotonin syndrome has been reviewed recently ${ }^{3}$ and is directed at discontinuation of all serotonergic medication with provision of supportive care as necessary. This may include muscular paralysis and ventilation when myoclonic activity involving thoracic muscles results in respiratory failure. Various pharmacological approaches have been tried with cyproheptadine (an antiserotonergic drug) being thought to be the most consistently effective agent.

My thanks to Dr T Battcock for allowing me to report this case, to Dr C Wulff for his help with EEG interpretation, and to Mrs C Evison for typing the manuscript.

1 Sternbach H. The serotonin syndrome. Am f Psychiatry 1991;148:705-13.

2 Bodner RA, Lynch T, Lewis L, et al. Serotonin syndrome. Neurology 1995;45:219-23.

3 Lane R, Baldwin D. Selective serotonin reuptake inhibitorinduced serotonin syndrome: review. F Clin Psychopharmacol 1997; 17:208-21.

4 Lejoyeux M, Finejre F, Adis J. The serotonin syndrome. Am f Psychiatry 1992;149:1410-1.

5 Dike G. Triphasic waves in serotonin syndrome (letter). $\mathcal{F}$ Neurol Neurosurg Psychiatry 1997;62:200.

6 Meynaar IA, Peeters AJ, Mulder AH, et al. Syndrome of inappropriate $\mathrm{ADH}$ secretion attributed to the serotonin re-uptake inhibitors, venlafaxine and paroxetine. Neth $\mathcal{F} M e d$ $1997 ; 50: 243-5$. 